\title{
Recherches participatives, recherches citoyennes... une clarification nécessaire
}

Depuis quelques années, les recherches participatives sont à l'honneur et présentées comme une des façons de rapprocher chercheurs et citoyens dans de nombreux domaines, alors que jusqu'à récemment elles exprimaient un type de recherche particulier dans le domaine des approches dites compréhensives. Notre dernier éditorial $(N S S, 20,4)$ s'appuyait sur l'implication de l'association NSS-Dialogues dans la nouvelle Alliance sciences société pour regretter l'absence d'expression de la société civile dans le processus d'élaboration de la future loi d'orientation de l'enseignement supérieur et de la recherche.

Nous nous réjouissons bien sûr, en tant que rédacteurs en chef d'une revue qui s'intitule Natures Sciences Sociétés, de la mise à l'agenda de telles préoccupations. Mais ne cachent-elles pas plusieurs visions bien différentes de ces relations entre chercheurs et citoyens et de la participation de ces derniers à la recherche?

Comme pour l'interdisciplinarité, on peut identifier plusieurs modalités de ces relations, différenciant finalités et formes d'implication des deux parties, si tant est que la «société » puisse être considérée comme univoque... On pourrait ainsi distinguer au moins trois postures.

La première, et c'est ce qui est revendiqué par l'Alliance évoquée ci-dessus, renvoie à la dimension institutionnelle de la recherche publique en l'invitant à une meilleure pratique démocratique: son rôle dans la société, l'élaboration de la programmation, l'identification des priorités, le suivi et l'évaluation des projets. Et pourtant, hormis peut-être dans le cas des acteurs industriels, la future loi d'orientation, qui fait tant référence et appel à l'innovation fait peu de cas des autres acteurs et de leurs innovations, qu'elles soient économiques, techniques, organisationnelles... Confondrait-on encore invention et innovation ? La transformation de l'invention en innovation ne se fait pas dans les laboratoires mais par son appropriation par des acteurs sociaux dans un monde fait de compétitions et de rapports de pouvoir... Ce sont ces alliances, entre recherche et société civile, qui génèrent le changement. Et cela pourrait effectivement se codifier, ainsi que cela se réalise dans les grands assessments conduits à l'échelle mondiale par la Banque mondiale et les agences des Nations unies. Le GIEC, le MEA ou $1^{\prime}$ IAASTD $^{1}$, dont nous vous avons déjà parlé à plusieurs reprises, associent par exemple, tant dans leur système de gouvernance que dans la pratique de l'expertise de base, des chercheurs, des ONG et des représentants des États.

Autre chose est d'enrôler des non-chercheurs dans des dispositifs de recherche afin de leur attribuer certaines tâches liées à leur expertise, à leur compétence, à leur pratique professionnelle ou, parfois, à l'image que l'on s'en fait. L'appel à la participation, qui va souvent de pair avec l'appel à l'interdisciplinarité, cache des réalités contrastées. La construction des problèmes d'environnement global a besoin de s'appuyer sur un « local », celuici souvent réinventé dans le monde du développement. De même, le mot d'ordre de développement durable exige la participation des populations locales. On peut comprendre alors l'intérêt porté aux savoirs locaux (profanes, indigènes, tacites...) en référence à des démarches inspirées de l'ethnoscience lors des négociations internationales sur la biodiversité. Un tel intérêt pour les compétences citoyennes n'est pas nouveau, surtout à plus petite échelle, si l'on songe par exemple aux baguages et recaptures d'oiseaux migrateurs ou aux inventaires de l'avifaune qui, depuis plusieurs décennies, reposent sur de vastes réseaux de bénévoles naturalistes, associant ainsi la démultiplication de la force de travail hors des laboratoires et l'intégration des connaissances expertes des ornithologues amateurs...

Enfin, c'est encore tout autre chose de créer les conditions de processus d'apprentissages croisés entre des chercheurs et leurs partenaires non chercheurs dans le cadre de ce qui est souvent qualifié de recherche-action, de recherche clinique, de recherche intervention, etc.,

1 GIEC : Groupe d'experts intergouvernemental sur l'évolution du climat; MEA: Millenium ecosystem assessment; IAASTD : International assessment of agricultural knowledge, science and technology for development. 
autant de termes dont on sait bien qu'ils ne sont pas équivalents. L'intégration des savoirs de ces partenaires dans le processus de recherche permet aussi bien de révéler des connaissances tacites que d'en produire de nouvelles issues directement de ces interactions. De telles recherches mobilisent des concepts mais avec l'objectif de fournir des connaissances pertinentes pour l'action (par ceux qui sont confrontés à une situation qui leur pose problème) et suffisamment heuristiques pour pouvoir être confrontées à d'autres situations (par les chercheurs engagés dans de telles collaborations). Certains parlent ainsi de transdisciplinarité dans un continuum entre connaissance et action. N'oublions pas que les problèmes traités sur le terrain ne sont jamais, tels quels, ceux des chercheurs, mais bien ceux de leurs partenaires : il y a ainsi toujours une double distance entre chercheur et praticien, entre leurs points de vue sur la réalité et entre la nature et le niveau d'élaboration des problèmes que les uns et les autres formulent ${ }^{2}$.

Exemplaires de ce point de vue, les recherches médicales se sont ouvertes à ces dimensions à la suite de l'organisation des malades du Sida et de leur revendication d'être associés (ou au moins leurs représentants dûment mandatés) à la programmation et à la définition des priorités de recherche, en rappelant aux chercheurs et au corps médical, qu'ils étaient, après tout, les premiers concernés ${ }^{3}$. Depuis, le curseur des collaborations entre patients et médecins, chercheurs ou cliniciens, s'est déplacé. On parle maintenant, dans le monde médical, de recherches translationnelles, et pas seulement à propos du sida, mais également dans d'autres domaines comme la cancérologie.
Depuis plusieurs années, nous avons publié dans NSS des textes portant sur ces questions. Le lecteur en trouvera dans ce numéro plusieurs qui illustrent cette diversité, de la prise en compte de la société civile, jusqu'aux situations de collaboration entre chercheurs et non-chercheurs, à propos de questions relatives à l'environnement, à la gestion de l'eau ou à l'organisation de l'espace rural.

Il nous a semblé utile, face à une certaine confusion qui semble régner dans cette nomenclature, d'essayer de clarifier la polysémie de l'expression « recherche participative » afin de mieux distinguer les dispositifs et les conditions de réalisation et d'intéressement aux résultats de ces formes de collaboration, qui ne sauraient être les mêmes dans les trois acceptions rappelées ci-dessus. N'oublions pas qu'à la différence de ce que sont les points de vue de la vie quotidienne, définis par les activités des sujets et leurs positions par rapport à une réalité ${ }^{4}$, les points de vue scientifiques se construisent au sein de disciplines, pourvoyeuses de paradigmes, de concepts et de méthodes, ce qui interpelle les chercheurs tout particulièrement lorsque, exerçant leur art dans des démarches collectives où les acteurs sont parties prenantes, ils s'engagent également sur la voie de l'interdisciplinarité.

\author{
Bernard Hubert, \\ Catherine Aubertin, \\ Jean-Paul Billaud
}

\footnotetext{
2 Darré J.-P., 1997. L'invention des pratiques dans l'agriculture. Vulgarisation et production locale de connaissance, Paris, éditions Karthala.

3 Rabeharisoa V., Callon M., 2002. The involvement of patients' associations in research, International Social Science Journal, Special issue: The Knowledge Society, 171, 57-65 ; Dodier N., 2003. Leçons politiques de l'épidémie de sida, Paris, éditions de l'EHESS.

4 Priéto L. J., 1975. Études de linguistique et de sémiologie générales, Genève, éditions Droz.
} 